\title{
Art and Science
}

\section{Sir Arnold Wolfendale}

\begin{abstract}
Science and art are two of the most significant facets of human endeavour, with astronomy occupying a key role within science. Despite C.P. Snow's Two Cultures, there are many common aspects and each has an influence on the other. A brief analysis is made of the relationship of art and science: the effect of art on science and science on art. Some outstanding problems are identified.
\end{abstract}

\section{Introduction}

At first sight, art and science are uneasy bed-fellows, the one (art) being largely subjective and its appreciation being in the eye of the beholder, whereas the other (science) being objective and its appreciation being universal. Some remarks are necessary about the latter, however, because of the significance of religion; this subject has great impact on art and some branches of science are still bedevilled by religious distortions. The scope of this present article is limited to concentrating on visual art, specifically painting, and on the physical sciences. The discussion falls into two parts: the influence of art on science and the influence of science on art.

\section{The Influence of Art on Science}

\subsection{The value of art in illustrations of scientific phenomena}

As soon as one embarks on an analysis of any topic the question of definition appears, and the present work is no exception. Visual art is here regarded as a pictorial attempt to get across certain ideas, not just of pure beauty, but of meaning. Thus, the graphical representation of data often has a pictorial value that aids one's appreciation. The value of colour, here, adds another dimension to appreciation and explanation. Figure 1 from radio astronomy is an example; colour adds a quantitative and well as a qualitative value to the presentation of experimental data.

Sir Arnold Wolfendale, 'Art and Science', eds. Nicholas Campion and Rolf Sinclair, Culture and Cosmos, Vol. 16 nos. 1 and 2, 2012, pp. 261-269. www.CultureAndCosmos.org 


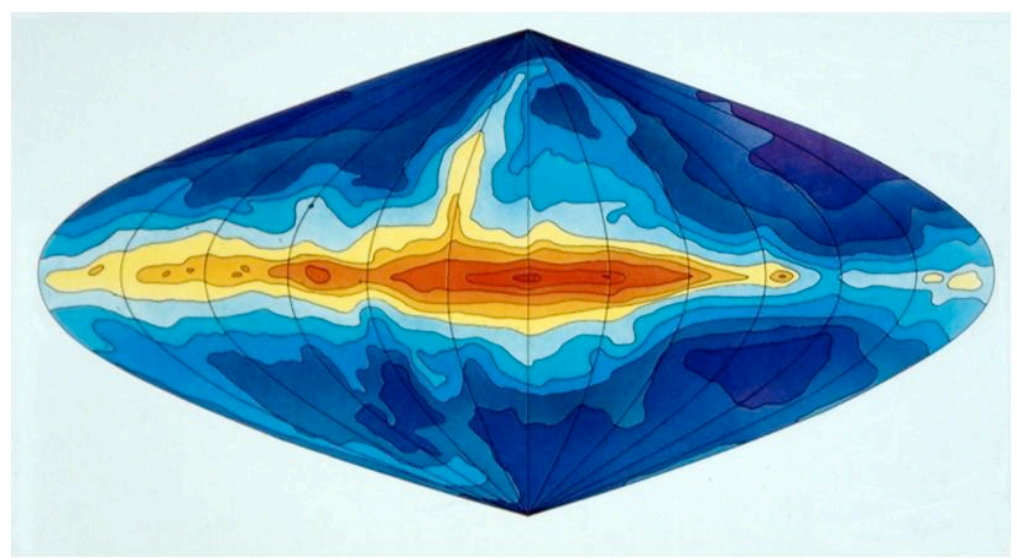

Figure 1. The sky at $100 \mathrm{MHz}$

Another example can be drawn from the early days of chemistry; the molecular modelling kits of the 1870 s allowed potentially valuable new molecules to be designed. The much more recent 'double-helix' model is perhaps the most important example.

\subsection{Art as a stimulus to the progress of science and technology}

From many examples one can choose horology as an example where, in the author's view, the emphasis of art on design led to the development of superb clocks and watches from the seventeenth century onwards. Harrison's 'H4' of 1759 (Figure 2) for which he eventually was effectively awarded the 1714 longitudinal Prize for navigation at sea, is a good example.

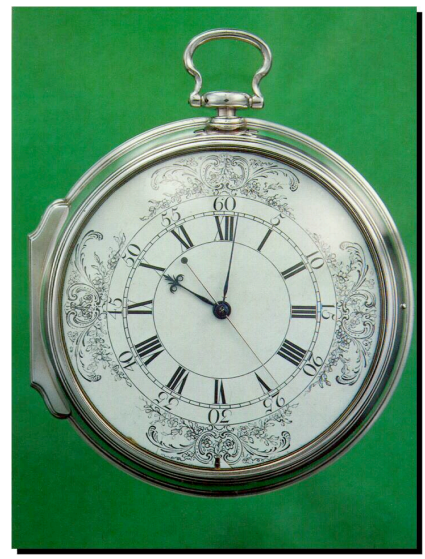

Figure 2. Harrison's 1759 watch 'H4' for navigation at sea

Culture and Cosmos 
The desire to construct an object of beauty as well as utility can be seen in the tracery of the engraving. Such ideas as simplicity and symmetry in design surely manifested themselves in the design of the clockwork, as well as the mounting. The influence of art on original discoveries, as distinct from stimulating improved design and construction, is less easy to discern. However, a personal example comes from Michelangelo's famous Sistine Chapel fresco depicting the Origin of Man (Figure 3).

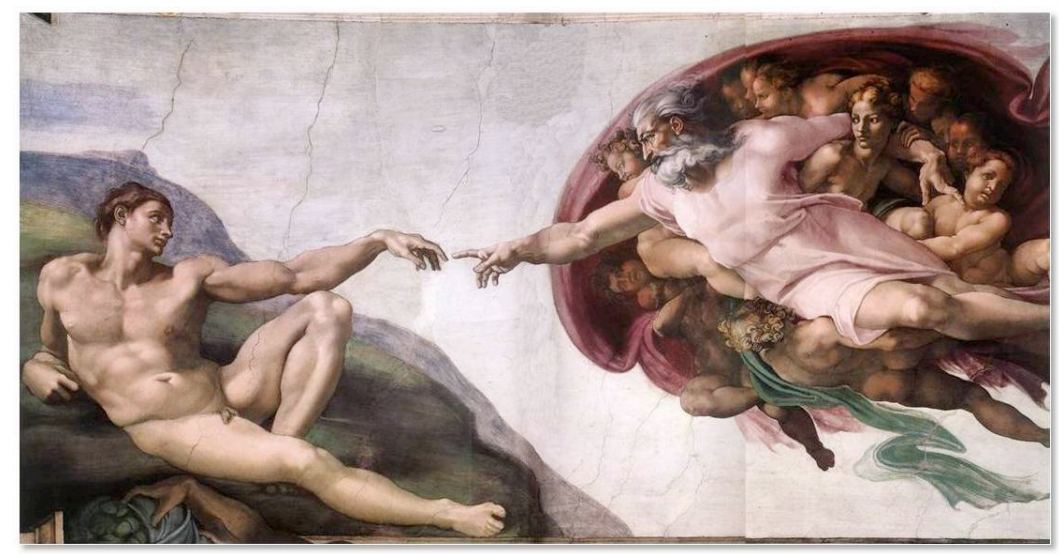

Figure 3. Michelangelo's Origin of Man

The electrifying 'Touch' made Tolya Erlykin and $\mathrm{I}^{1}$ go back to Miller's idea involving the creation of life by lightning in the early Earth. $^{2}$ A full theory involving the (variable) creation of lightning strikes by cosmic rays was worked out. It might even be right! (Figure 4).

\footnotetext{
${ }^{1}$ A.D. Erlykin and A.W. Wolfendale, 'Long term time variability of cosmic rays and possible relevance to the development of life on Earth', Surveys in Geophysics, Vol. 31, Issue 4 (July 2010): p. 383.

${ }^{2}$ S.L. Miller, 'A production of amino-acids under possible primitive earth conditions', Science 117(3046) (May 15, 1953): p. 528.
} 


\section{Art and Science}

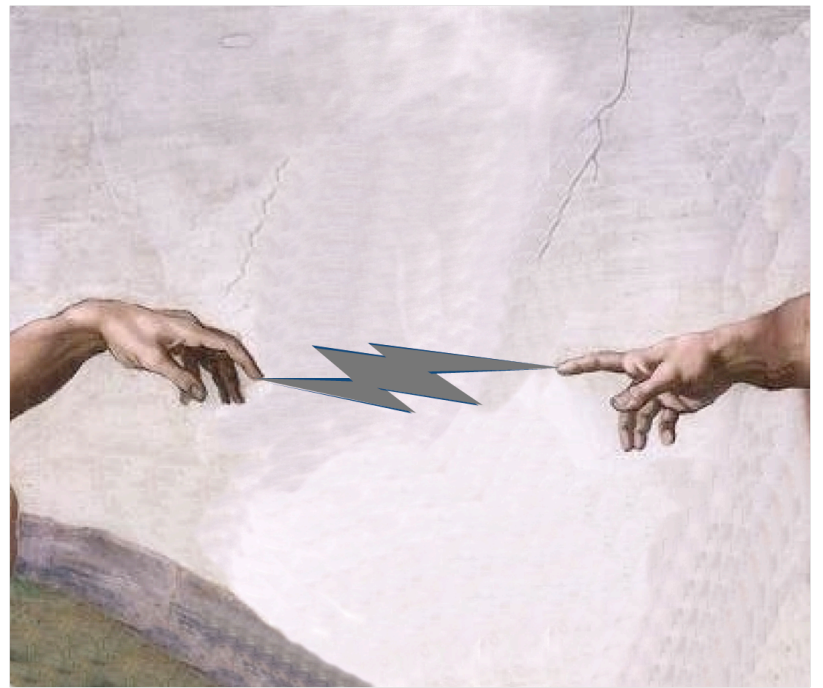

Figure 4. A variant of the touch

Before leaving the influence of art on science there are some scientific phenomena that are very difficult (impossible?) to understand. Artistic representation can help considerably. Figure 5 is a good example, drawn from the field of quantum mechanics. The apparent passage of an atomic particle around both sides of an obstacle is a phenomenon easy to describe mathematically but less easy pictorially.

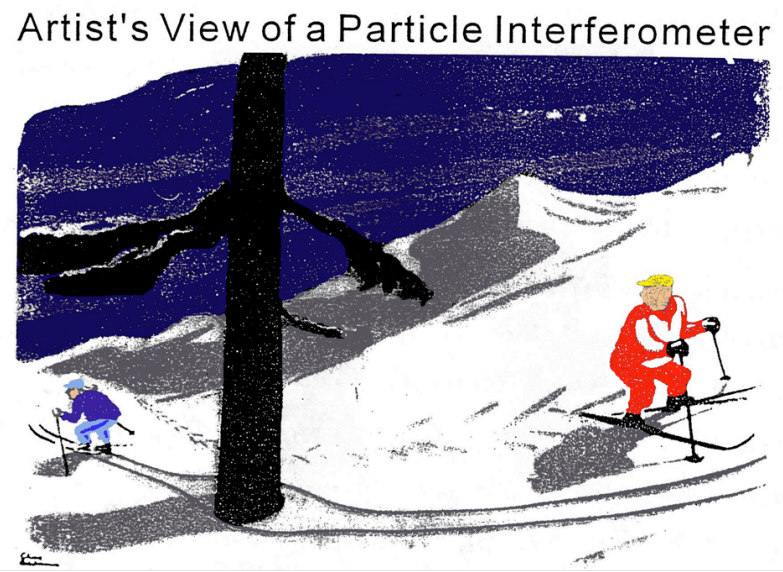

Figure 5.

Culture and Cosmos 


\section{The Influence of Science on Art}

\subsection{New materials}

By 'science' we include technology and immediately the question of materials comes up, from rock indentations to acrylic paint. Just a few examples will be given. It seems that Impressionism owed much of its impact to the vibrant hues of the new materials. Seurat's work is a good example (Figure 6).

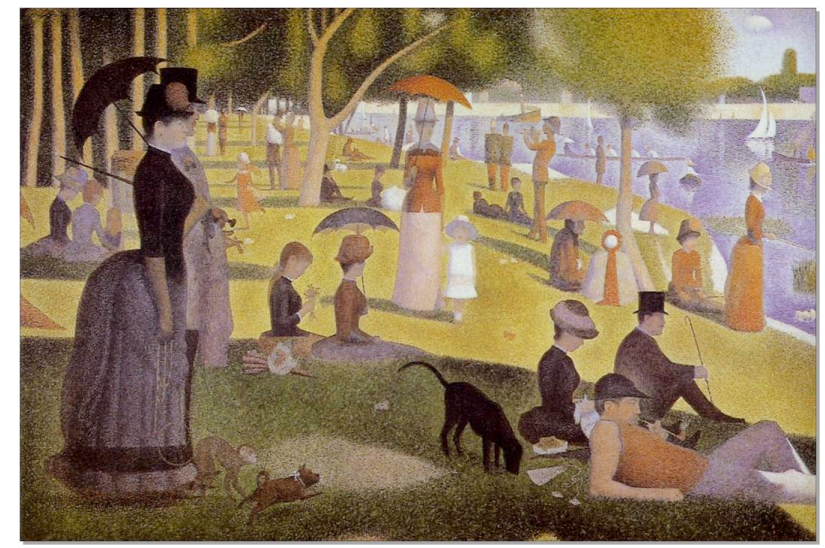

Figure 6. Sunday afternoon on the island of La Grande Jatte, Georges Seurat

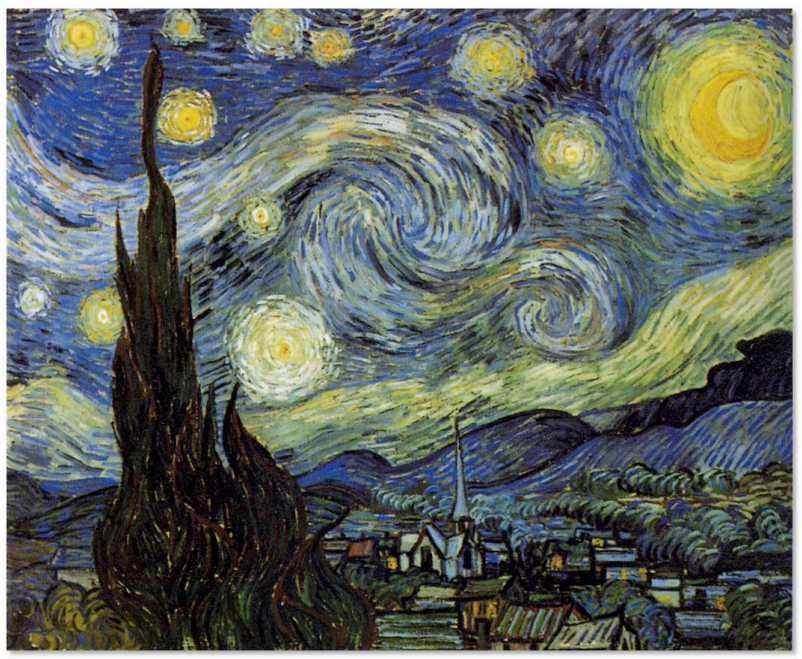

Figure 7. The Starry Night, Vincent Van Gogh 


\section{Art and Science}

Van Gogh's 'Starry Night' (Figure 7) is another. It is interesting that Van Gogh's painting of spirals in the Heavens may be based on the by-then-known fact that our 'nearby' ( 2 light years away) galaxy, Andromeda, has a smaller accompanying one.

\subsection{Perspective and Cubism}

'Early' paintings were very often flat and stylised, with little apparent depth. The introduction of perspective was a great step forward. To my way of thinking Cubism (e.g., Figure 8) is a development in the same direction - a half-way home between realism and subjectivism.

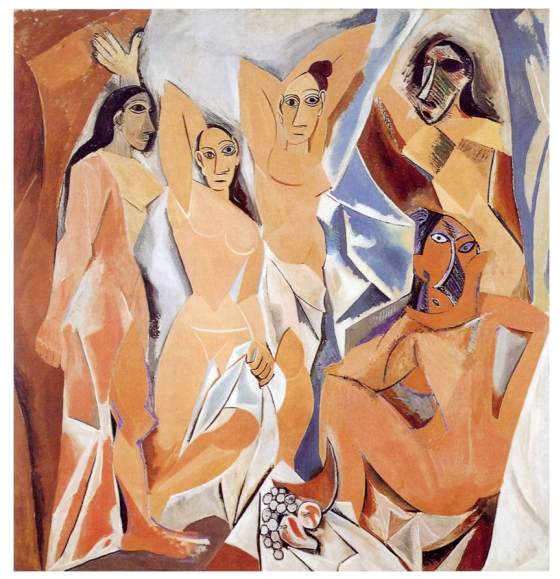

Figure 8. Les Demoiselles d'Avignon, Pablo Picasso

What might be called 'Exaggerated Perspective', which conveys a powerful image, comes from the work of Tom McGuinness (Figure 9).

Figure 9. Harvey Manrider, Tom McGuinness

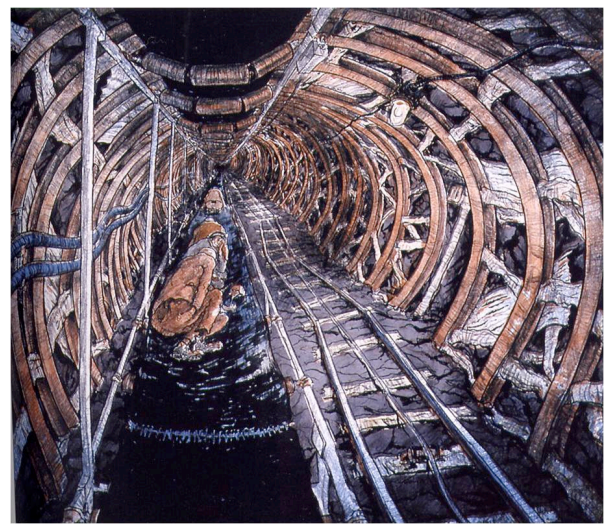

Culture and Cosmos 
One of my favourite artists is Nitin Nangre of Pune, India (Figure 10 ), whose use of a subdued cubism is brilliant.

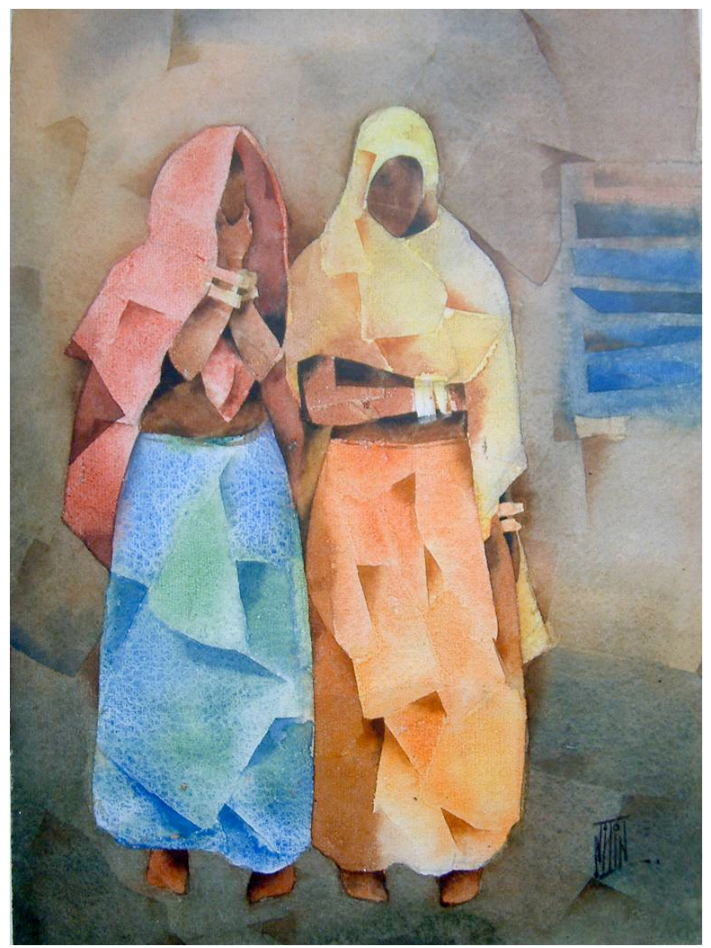

Figure 10. Local women, Nitin Nangre

\subsection{Influence of science on the artists themselves}

Henri Becquerel's discovery in the 1890 s that matter was 'radioactive' and could decay had a profound effect on the budding Wassily Kandinsky in Moscow. He wrote:

In my soul the decay of the atom was the same as the decay of the whole world. Suddenly the sturdiest walls collapsed. Everything became uncertain, unsteady, and soft. It would not have amazed me, if a stone had melted into air before me and become invisible. $^{3}$

${ }^{3}$ Cited in Wassily Kandinsky 1866-1944: a Revolution in Painting, ed. Hajo Düchting (Los Angeles: Taschen, 2000), p. 10. 


\section{Art and Science}

Kandinsky, who went on to found an important school of modern art in Germany, also wrote:

When religion, science and morality are shaken...... when the external supports threaten to collapse, man's gaze turns from the external world towards himself, ${ }^{4}$

From Kandinsky's many impressive works, Figure 11 involves, to me, much scientific input.

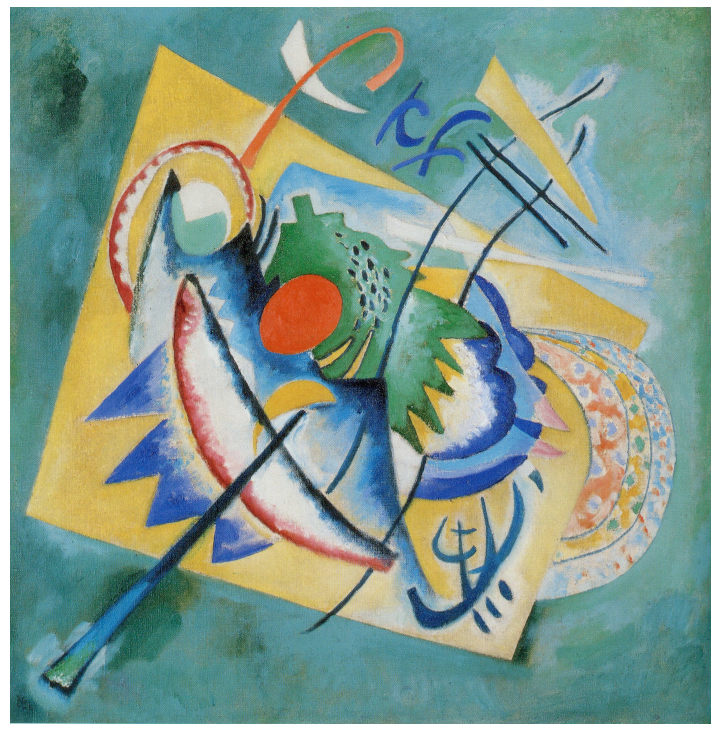

Figure 11. Red Oval, Wassily Kandinsky

\subsection{The origin of the universe}

A popular topic for illustration by modern artists is 'the origin of the universe'. The depiction of the Big Bang is a fine challenge. Of many such, Figure 12 by Jozef Krivanek is a good example.

\footnotetext{
${ }^{4}$ Wassily Kandinsky, Concerning the Spiritual in Art, trans. M.T.H. Sadler (New York: Dover Publications, 1977), p. 14.
}

Culture and Cosmos 


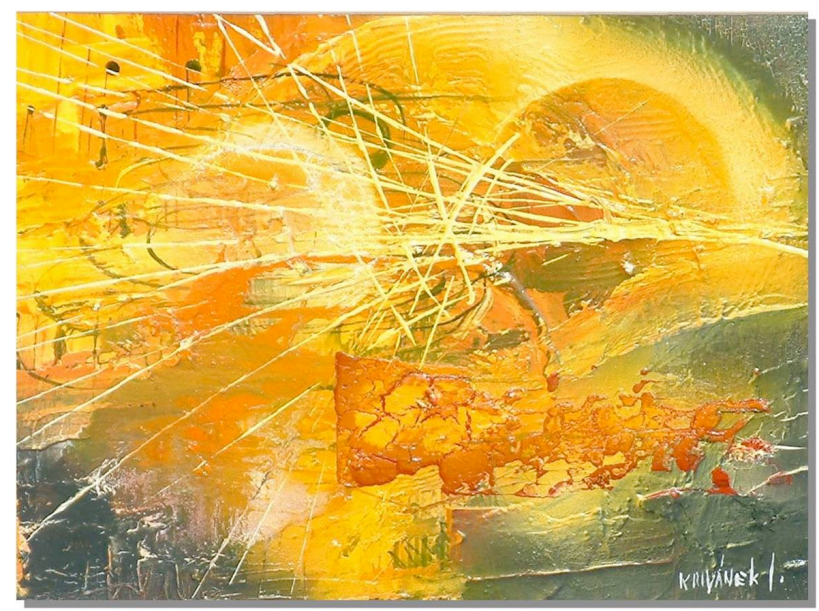

Figure 12. The origin of the universe, Jozef Krivanek

To me, however, I prefer to turn the picture through a right angle; it then represents a shower of atomic particles initiated in the atmosphere by a very high-energy cosmic ray!

\section{Problems to be solved}

Of the many questions raised by a discussion of the inter-relation of science and art, I mention just two. The first is the claim by David Hockney of the 'use of optics' by the old masters. The hypothesis is that lenses and prisms were used by some to throw images onto the artist's drawing paper so that outlines at least could be drawn. Hockney makes a good case; certainly there were such aids available, but the extent to which they were used is not certain. An added complication was the frequent use of juniors to do at least some of the work on what were often large and complicated canvasses.

The second is rather more cerebral: the extent to which advances in science led to changes in the direction of art. The example of perspective has been mentioned but that was an obvious development, the object of which was the need to make paintings more realistic. What about the advent of Impressionism? The role of developments in modern physics in Kandinsky's 'evolution' has been mentioned. To what extent were the quite fundamental changes in ideas in the late nineteenth and early twentieth centuries - which led to the uncertainty principle and other strange ideas - responsible for the equally strange concepts in impressionism? The jury is still out. 